\title{
Saccharomyces boulardii ameliorates clarithromycin- and methotrexate- induced intestinal and hepatic injury in rats
}

\author{
Deniz Güney Duman ${ }^{1 *}$, Zarife Nigâr Özdemir Kumral², Feriha Ercan ${ }^{3}$, Mustafa Deniz ${ }^{4}$, Güray Can ${ }^{5}$ \\ and Berrak Çăglayan Yeğen ${ }^{2}$ \\ ${ }^{1}$ Department of Gastroenterology, School of Medicine, Saglik Bakanligi Marmara Universitesi Pendik E.A.H., Mimar Sinan \\ Caddesi 41, Üst Kaynarca Pendik, 34899 Istanbul, Turkey \\ ${ }^{2}$ Department of Physiology, School of Medicine, Marmara University, Istanbul, Turkey \\ ${ }^{3}$ Department of Histology and Embryology, School of Medicine, Marmara University, Istanbul, Turkey \\ ${ }^{4}$ Department of Physiology, School of Medicine, Canakkale Onsekiz Mart University, Canakkale, Turkey \\ ${ }^{5}$ Department of Gastroenterology, School of Medicine, Trakya University, Edirne, Turkey \\ (Submitted 22 June 2012 - Final revision received 26 September 2012 - Accepted 16 October 2012 - First published online 2 January 2013)
}

\begin{abstract}
Saccharomyces boulardii is a probiotic used for the prevention of antibiotic-associated diarrhoea. We aimed to investigate whether $S$. boulardii could alter the effects of clarithromycin (CLA) and methotrexate (MTX) on oro-caecal intestinal transit and oxidative damage in rats. Rats were divided into two groups receiving a single dose of MTX $(20 \mathrm{mg} / \mathrm{kg})$ or CLA (20 mg/ $\mathrm{kg}$ per d) for $1 \mathrm{keek}$. Groups were treated with either saline or $S$. boulardii $(500 \mathrm{mg} / \mathrm{kg})$ twice per d throughout the experiment. The control group was administered only saline. Following decapitation, intestinal transit and inflammation markers of glutathione (GSH), malondialdehyde and myeloperoxidase were measured in intestinal and hepatic tissues. CLA and MTX increased intestinal transit, while $S$. boulardii treatment slowed down CLA-facilitated transit back to control level. Both MTX and CLA increased lipid peroxidation while depleting the antioxidant GSH content in the hepatic and ileal tissues. Conversely, lipid peroxidation was depressed and GSH levels were increased in the ileal and hepatic tissues of $S$. boulardii-treated rats. Increased ileal neutrophil infiltration due to MTX and CLA treatments was also reduced by $S$. boulardii treatment. Histological analysis supported that $S$. boulardii protected intestinal tissues against the inflammatory effects of both agents. These findings suggest that $S$. boulardii ameliorates intestinal injury and the accompanying hepatic inflammation by supporting the antioxidant state of the tissues and by inhibiting the recruitment of neutrophils. Moreover, a preventive effect on MTXinduced toxicity is a novel finding of $S$. boulardii, proposing it as an adjunct to chemotherapy regimens.
\end{abstract}

Key words: Saccharomyces boulardii: Clarithromycin: Methotrexate: Intestinal toxicity: Hepatic toxicity

Probiotics are viable micro-organisms that confer health benefits to the host when administered in adequate amounts. They have been used to treat several acute infectious and chronic intestinal diseases ${ }^{(1)}$. Saccharomyces boulardii is a probiotic yeast that has been shown to be effective in the prevention of antibiotic-associated diarrhoea (AAD) ${ }^{(2)}$. It exerts trophic effects, both in the mucosa and the endoluminal fluid of the small intestine, which appears to be mediated by the endoluminal release of polyamines ${ }^{(3)}$. As secretion of IgA and polymeric Ig receptors into the lumen of the small intestine impairs the attachment of micro-organisms and external antigens to intestinal epithelial cells, the proliferation of pathogens in the gut lumen is thus prevented ${ }^{(4)}$.
In a mouse model of inflammatory bowel disease, $S$. boulardii treatment was shown to inhibit inflammatory bowel disease by suppressing $\mathrm{CD}^{+}{ }^{+}$T-cell number and interferon- $\gamma$ production within the colon, suggesting that both humoral and cellular immune defences are involved in the beneficial effects of $S$. boulardii on $\mathrm{AAD}^{(1)}$. Recent data support the efficacy of $S$. boulardii in gastrointestinal inflammatory conditions, including bacterial infections and inflammatory bowel disease, through modulation of host pro-inflammatory responses by controlling inflammation at different levels, such as the NF- $\mathrm{B}$ and the mitogen-activated protein kinase pathways ${ }^{(5,6)}$. However, the effects of probiotics on oro-caecal motility or

Abbreviations: AAD, antibiotic-associated diarrhoea; CLA, clarithromycin; GSH, glutathione; MDA, malondialdehyde; MPO, myeloperoxidase;

MTX, methotrexate.

*Corresponding author: D. G. Duman, email gduman@marmara.edu.tr 
gastrointestinal antioxidant capacity in inflammatory models have not been described before.

$\mathrm{AAD}$ is caused by antibiotics used to treat infections by enteric pathogens via the direct effects on the intestinal mucosa or due to the metabolic consequences of reduced concentrations of faecal flora ${ }^{(7)}$. However, neither mechanism is clearly established as the cause of AAD. Although the frequency of $\mathrm{AAD}$ varies among antibacterial agents, diarrhoea occurs in $7.5 \%$ of patients treated with clarithromycin $(C L A)^{(8)}$. CLA, a semi-synthetic macrolide antibiotic that inhibits bacterial protein synthesis, is clinically active against Gram-positive and Gram-negative cocci, Gram-negative bacilli (primarily Haemophilus influenzae, Legionella species, Moraxella catarrhalis, Campylobacter jejuni, Bordatella pertussis and Helicobacter pylori) ${ }^{(9)}$. It undergoes extensive hepatic metabolism, mainly by hydroxylation, and there is a substantial first-pass effect. Unchanged CLA and its metabolites are eliminated in the faeces and urine ${ }^{(9)}$. Despite the extensive use of CLA in the treatment of skin, soft tissue, upper and lower respiratory tract infections, sexually transmitted Chlamydia trachomatis infection and as a component of anti- $H$. pylori regimens, AAD and hepatotoxicity frequently limit its use $\mathrm{e}^{(10)}$.

Methotrexate (MTX), a folic acid antagonist, is widely used as a cytotoxic chemotherapeutic agent for leukaemia and other malignancies. MTX inhibits dihydrofolic acid reductase, and thus interferes with DNA synthesis, repair and cellular replication $^{(11)}$. As the cytotoxic effect of MTX is not selective for cancer cells, normal tissues that have a high rate of proliferation, including the haematopoietic cells of the bone marrow and the actively dividing cells of the gut mucosa, are more susceptible to toxicity. Additionally, two rat studies have shown increased intestinal transit with parenteral $\operatorname{MTX}^{(12,13)}$, though the relevant data are lacking in human subjects. Thus, the efficacy of the agent is often limited by severe hepatic and intestinal mucosal damage.

The purpose of the present study was to elucidate the putative protective effects of $S$. boulardii on oxidative damage induced by two different mechanisms, namely, metabolic alteration of faecal flora by CLA and direct cellular toxicity by MTX. We also aimed to study the impact of the yeast therapy on AAD-induced and MTX-induced diarrhoea.

\section{Materials and methods}

\section{Animals}

Adult male Sprague-Dawley rats (250-320g) supplied by the Marmara University Animal Centre (DEHAMER) were housed individually in a light- and temperature-controlled room on a $12 \mathrm{~h}$ light $-12 \mathrm{~h}$ dark cycle, where the temperature $\left(22 \pm 2^{\circ} \mathrm{C}\right)$ and relative humidity $(65-70 \%)$ were kept constant. The animals were fed a standard pellet laboratory chow, and food was withdrawn overnight before emptying experiments, but free access to water was allowed. Experiments were approved by the Marmara University Animal Care and Use Committee.

\section{Administration of drugs}

Rats either received a single intraperitoneal injection of MTX (20 mg/kg; Onco-Tain, Faulding Pharmaceutics Plc) or they were administered daily with CLA (20 mg/kg per d; Deva İlaç Sanayii) for $7 \mathrm{~d}$ by an orogastric catheter under brief diethyl ether anaesthesia. Following the single MTX injection or accompanying the CLA administration, rats were also treated with either saline or S. boulardii (Reflor ${ }^{\circledR}$, Biocodex; $500 \mathrm{mg} / \mathrm{kg}$ twice per d) by an orogastric catheter for seven consecutive days. S. boulardii is available as a lyophilised preparation in Turkey, which is stable for over 1 year at room temperature when protected from moisture and maintains high viability counts over prolonged periods ${ }^{(6)}$. The viability analysis of the probiotic showed $2.5 \times 10^{9}$ living cells per $250 \mathrm{mg}$ sachet, as provided by the manufacturer. The dose selection of the test drugs was made according to the previous in vivo rat studies ${ }^{(13-16)}$. In control rats, following a single dose of saline injection, saline was administered orally for $7 \mathrm{~d}$. Groups consisted of seven to nine rats.

\section{Measurement of intestinal transit}

All rats were fasted overnight and intestinal transit studies were performed by giving $1 \mathrm{ml}$ of a mixture of Arabic gum (gum Arabic from Acacia tree, Sigma Chemical) and activated charcoal through an intraduodenal catheter on the 8th day of the experiment at 08.00 hours $^{(17)}$. After $20 \mathrm{~min}$, rats were killed by decapitation, the abdomen was opened and ligatures were made around the pylorus and ileocaecal valve. The small intestine was dissected and freed from its mesentery, with its continuity retained. The intestine was then measured by laying it longitudinally. To avoid movement of intraluminal contents, the intestine was not stretched. The total length of the small bowel and the length of small bowel filled with the black meal were recorded. Intestinal transit index (\%) was expressed as the fraction of the total length of the small bowel filled with the black material.

\section{Measurement of tissue myeloperoxidase activity}

Tissue-associated myeloperoxidase (MPO) activity is frequently utilised to estimate tissue neutrophil accumulation in inflamed tissues. The method of assay of MPO activity in the present study was similar to that previously described by others $^{(18)}$. The ileum and liver tissue samples $(0 \cdot 2-0.3 \mathrm{~g})$ were homogenised in ten volumes of ice-cold potassium phosphate buffer $\left(50 \mathrm{~mm}_{2} \mathrm{~K}_{2} \mathrm{HPO}_{4}, \mathrm{pH}\right.$ 6.0) containing hexadecyltrimethylammonium bromide $(0 \cdot 5 \%, \mathrm{w} / \mathrm{v})$. The homogenate was centrifuged at $41400 \mathrm{~g}$ for $10 \mathrm{~min}$ at $4{ }^{\circ} \mathrm{C}$, and the supernatant was discarded. The pellet was then rehomogenised with an equivalent volume of $50 \mathrm{~mm}-\mathrm{K}_{2} \mathrm{HPO}_{4}$ containing $0.5 \%(\mathrm{w} / \mathrm{v})$ hexadecyltrimethylammonium bromide and 10 mM-EDTA (Sigma). MPO activity was assessed by measuring the $\mathrm{H}_{2} \mathrm{O}_{2}$-dependent oxidation of $\mathrm{O}$-dianizidine. $2 \mathrm{HCl}$. One unit of enzyme activity was defined as the amount of MPO present per $g$ of tissue weight that caused a change in absorbance of $1.0 / \mathrm{min}$ at $460 \mathrm{~nm}$ and $37^{\circ} \mathrm{C}$. 


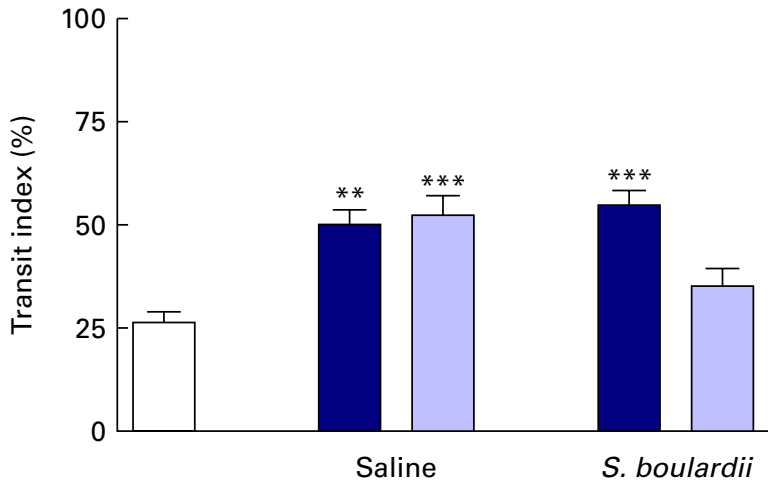

Fig. 1. Intestinal transit index (\%) in orally Saccharomyces boulardii- or salinetreated rats that received either methotrexate $(\square)$ injection or daily clarithromycin ( $\square$ ) by oral administration. Values are means, with standard errors represented by vertical bars. Mean values were significantly different compared with the saline-treated control ( $\square$ ) group: ${ }^{\star \star} P<0.01,{ }^{\star \star \star} P<0.0001$. (A colour version of this figure can be found online at http://www.journals.cambridge.org/bjn).

\section{Determination of lipid peroxidation (malondialdehyde)} and glutathione levels

Ileal and hepatic tissue samples were homogenised in a $10 \mathrm{ml}$ volume of ice-cold $10 \%$ trichloroacetic acid, in an Ultra Turrax tissue homogeniser. Homogenised tissue samples were centrifuged at $3000 \mathrm{rpm}$ for $15 \mathrm{~min}$ at $4^{\circ} \mathrm{C}$. The supernatant was removed and recentrifuged at $15000 \mathrm{rpm}$ for $8 \mathrm{~min}$. Glutathione (GSH) measurements were performed using a modification of the Ellman procedure ${ }^{(19)}$. Lipid peroxidation was quantified by measuring the formation of thiobarbituric acidreactive substances, as described previously ${ }^{(20)}$. Lipid peroxide levels were expressed in nmol malondialdehyde (MDA) per g tissue.

\section{Histopathological preparation and analysis}

For the light microscopic investigations, tissue specimens from the ileum were fixed with $10 \%$ formaldehyde and processed routinely for embedding in paraffin. Approximately, $5 \mu \mathrm{m}$ thick tissue sections were stained with haematoxylin and eosin and examined under an Olympus BX51 photomicroscope. Microscopic scoring was done by an experienced histologist, who was unaware of the treatments. Scores were given as 0 , none; 1 , mild; 2 , moderate; 3 , severe for each criterion, using the semi-quantitative scale as follows: (1) degeneration of surface and crypt epithelium; (2) degeneration of villus structure; (3) inflammatory cell infiltration. The microscopic score of the ileum was calculated as the sum of the scores given to each criterion, and at least five microscopic areas were examined to score each specimen. The maximum score that could be given was ' 9 '(21).

\section{Statistical analysis}

All data are expressed as means with their standard errors. Each group consisted of seven to nine rats. Groups of data were compared with an ANOVA followed by Tukey's multiple comparison tests, and for pair-wise comparisons, Student's $t$ test and Mann-Whitney $U$ test were used. Results were considered significant when $P$ was less than 0.05. Calculations were made using GraphPad Prism 3.0 (GraphPad Software).

\section{Results}

At the end of the experiment, all the rats in the groups had survived and no significant differences were observed among the weights of the animals.

\section{Effect of Saccharomyces boulardii on intestinal transit}

MTX and CLA significantly facilitated intestinal transit by nearly 2-fold in the saline-treated groups $(P<0.01$ and $<0.001$; Fig. 1). S. boulardii administration had no significant effect on increased intestinal transit due to MTX treatment. Nevertheless, S. boulardii depressed CLA-induced rapid intestinal transit, but was not statistically different from the transit index of the control group $(P>0 \cdot 05)$.

\section{Ileal and hepatic malondialdehyde and glutathione levels}

MDA levels determined in the ileum and liver tissues were found to be significantly higher in both the saline-treated MTX and CLA groups, as compared with those in the tissues of the control group $(P<0 \cdot 01$; Fig. 2(a) and (b)). In the ileum, $S$. boulardii administration significantly decreased the MTXor the CLA-associated lipid peroxidation $(P<0 \cdot 01)$. In the liver, $S$. boulardii was not effective on MTX-associated MDA elevation, but it depressed hepatic MDA of the CLA-treated group significantly $(P<0 \cdot 01)$.

The levels of the major cellular antioxidant GSH in both tissues were significantly decreased in the saline-treated MTX and CLA groups $(P<0 \cdot 01-0 \cdot 001$; Fig. 3(a) and (b)) Treatment with $S$. boulardii reduced the reductions in GSH
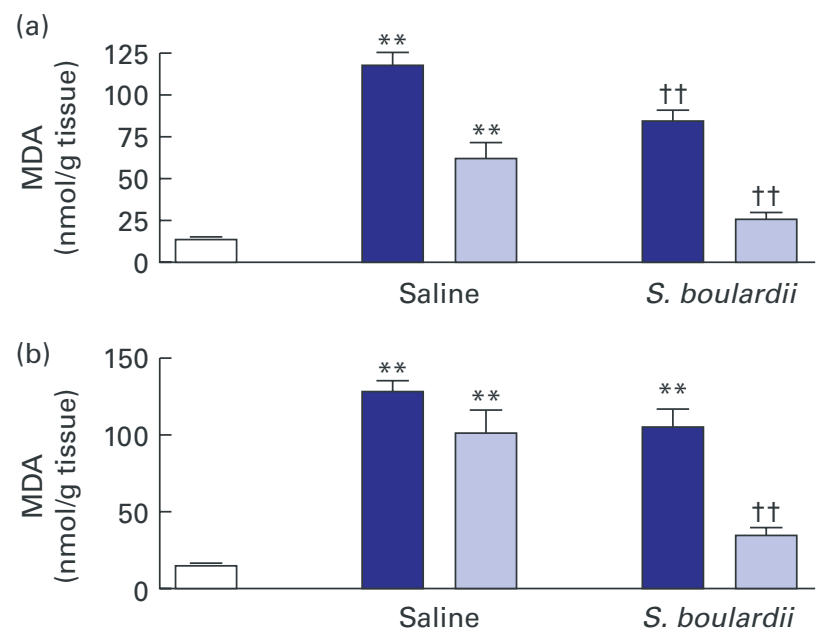

Fig. 2. Malondialdehyde (MDA) levels in the (a) ileum and (b) liver of the orally Saccharomyces boulardii- or saline-treated rats that received either methotrexate $(\square)$ injection or daily clarithromycin $(\square)$ by oral administration. Values are means, with standard errors represented by vertical bars. ${ }^{* *}$ Mean value was significantly different compared with the saline-treated control $(\square)$ group $(P<0.01)$. †† Mean value was significantly different compared with the respective saline-treated group $(P<0.01)$. (A colour version of this figure can be found online at http://www.journals.cambridge.org/bjn). 

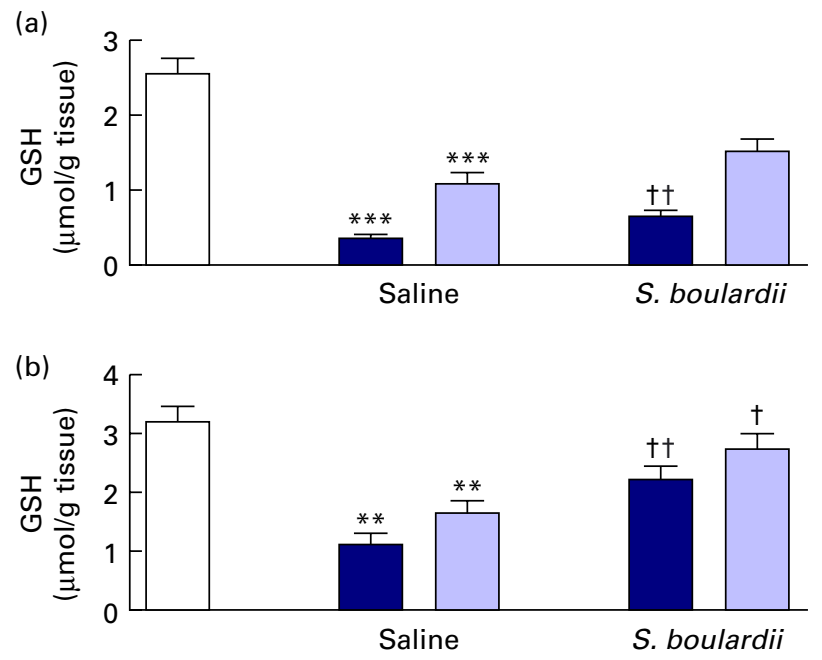

Fig. 3. Glutathione (GSH) levels in the (a) ileum and (b) liver of the orally Saccharomyces boulardii- or saline-treated rats that received either methotrexate ( $\square$ ) injection or daily clarithromycin ( $\square$ ) by oral administration. Values are means, with standard errors represented by vertical bars. Mean values were significantly different compared with the saline-treated control ( $\square$ ) group: ${ }^{*} P<0.01 ;{ }^{* *} P<0.001$. Mean values were significantly different compared with the respective saline-treated group: $\dagger P<0.05$; $\dagger \dagger P<0.01$. (A colour version of this figure can be found online at http://www.journals.cambridge.org/bjn)

content in the hepatic and ileal tissues of both the MTX- and the CLA-treated groups $(P<0 \cdot 05-0 \cdot 01)$.

\section{Tissue myeloperoxidase activity}

MPO activities in the ileal tissues were increased in the salinetreated MTX and CLA groups $(P<0 \cdot 001 ;$ Fig. 4(a)), indicating recruitment of neutrophils to the inflamed ileum. $S$. boulardii administration reduced both the MTX- and CLA-induced elevation in MPO activity in the ileal tissue $(P<0.001$ and $P<0.01)$. The MTX-induced increase in the hepatic MPO activity $(P<0 \cdot 001$, Fig. 4(b)) was depressed in the $S$. boularditreated group $(P>0.05)$. However, CLA treatment with or without $S$. boulardii had no impact on hepatic MPO activity.

\section{Histopathological results}

The light microscopic findings of the small-bowel mucosa were entirely normal in the control group. By contrast, histological analysis revealed the presence of MTX-induced damage in the ileum (Fig. 5). In the saline-treated MTX group, the ileal mucosa showed mild degeneration of the surface epithelium, flattened villi and severe inflammatory cell infiltration. In the MTX group treated with the probiotic, the ileum showed mild degeneration in the surface epithelium and mild inflammation. Severe degeneration in the surface epithelium and villi, and severe inflammation in the salinetreated CLA group were replaced with mild degeneration in the surface epithelium and moderate inflammation in the $S$. boulardii-treated CLA group. Histopathological scores of the ileum showed that $S$. boulardii treatment reduced the degeneration of the surface and crypt epithelium, the villus structure and depressed inflammatory cell infiltration of both the MTX and CLA-treated groups $(P<0 \cdot 05$, Fig. 6).

\section{Discussion}

The present findings revealed that CLA and MTX increased intestinal transit significantly, as compared with the control rats, while $S$. boulardii treatment slowed down CLA-facilitated transit back to control level. The results showed that both MTX and CLA increased lipid peroxidation of the ileal and hepatic tissues, along with depletion of the antioxidant GSH content in both tissues. Moreover, antioxidant GSH levels were increased in the ileal and hepatic tissues of $S$. boularditreated rats, and lipid peroxidation in these tissues was depressed. Accordingly, increased ileal neutrophil infiltration due to the pro-inflammatory MTX and CLA treatments was also reduced by $S$. boulardii treatment. In addition, histological analysis supported that $S$. boulardii significantly protected the intestinal tissues against the inflammatory effects of both agents. These findings suggest that $S$. boulardii ameliorates intestinal injury and the accompanying hepatic inflammation by supporting the antioxidant state of the tissues and inhibiting recruitment of pro-oxidant neutrophils to the tissues.

The macrolide group antibiotic CLA has a stimulatory effect on gut motility because it has a fourteen-membered ring like erythromycin, which stimulates gastroduodenal motility through the activation of motilin receptors ${ }^{(22,23)}$. Although having no direct action on motilin receptors, the ability of $S$. boulardii to counteract the disturbed motility induced by CLA could be explained by the beneficial effects of $S$. boulardii on altered gut flora. Within the intestinal lumen, $S$. boulardii may restore motility by suppressing the inflammatory state, as demonstrated in the present study. Since it is metabolised by cytochrome $\mathrm{P} 450$ isoenzymes ${ }^{(24)}$, asymptomatic

(a)
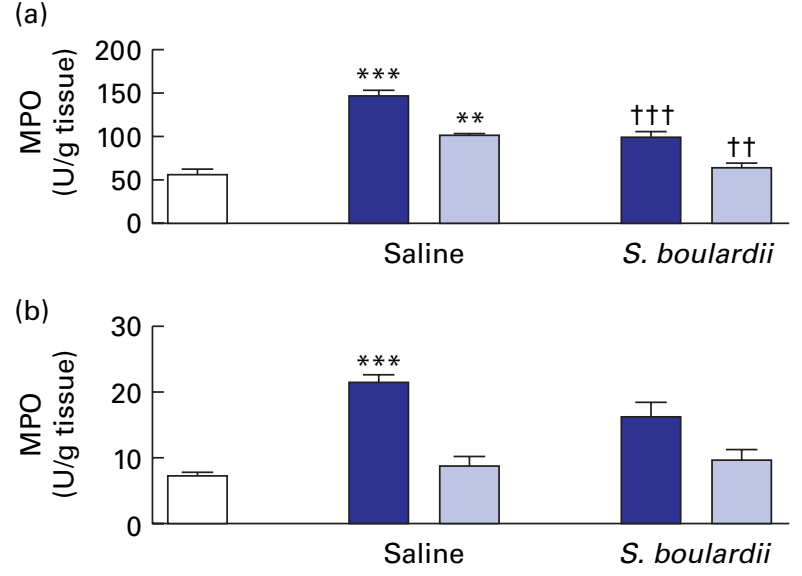

Fig. 4. Myeloperoxidase (MPO) activity in the (a) ileum and (b) liver of the orally Saccharomyces boulardii- or saline-treated rats that received either methotrexate $(\square)$ injection or daily clarithromycin $(\square)$ by oral administration. Values are means, with standard errors represented by vertical bars. Mean values were significantly different compared with the saline-treated control ( $\square$ ) group: ${ }^{\star \star} P<0.01 ;{ }^{\star \star \star} P<0.001$. Mean values were significantly different compared with the respective saline-treated group: $\uparrow \dagger P<0.01 ; \dagger \dagger \uparrow P<0.001$. (A colour version of this figure can be found online at http://www.journals. cambridge.org/bjn). 

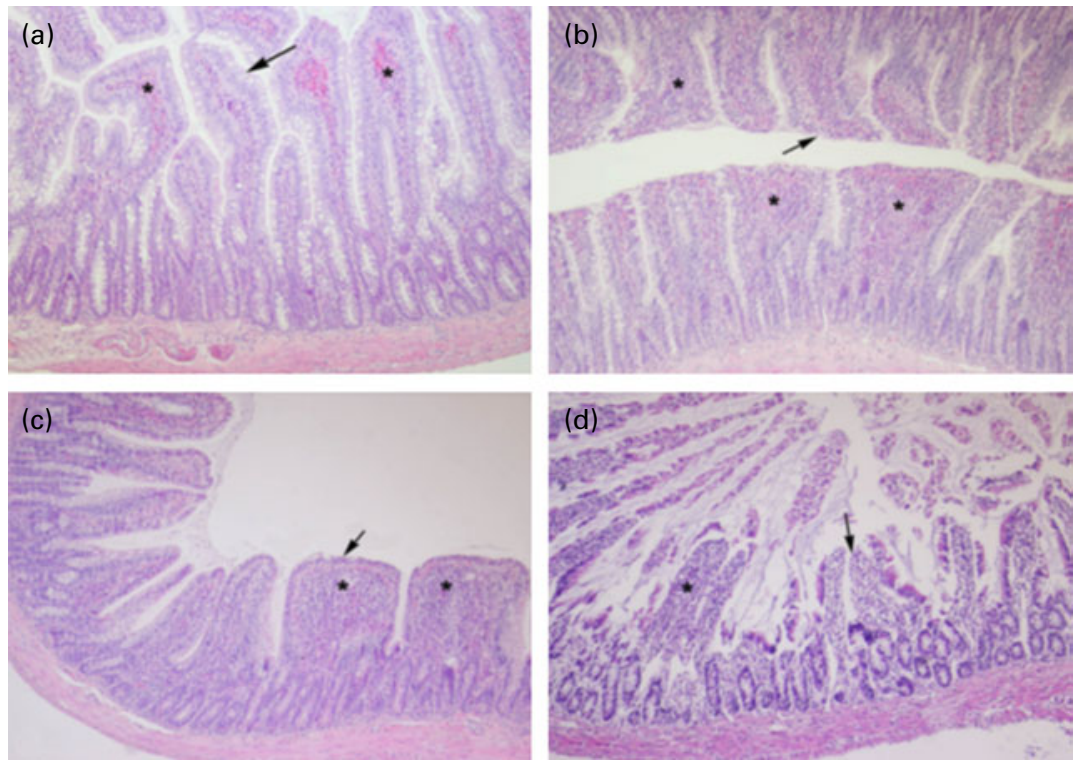

Fig. 5. Micrographs of ileal tissues. (a) Saccharomyces boulardii-treated methotrexate (MTX) group: mild degeneration in the surface epithelium ( $\rightarrow$ ), mild inflammatory cell infiltration $\left({ }^{\star}\right)$. (b) Saline-treated MTX group: mild degeneration in the surface epithelium $(\rightarrow)$, severe inflammatory cell infiltration $\left(^{\star}\right)$. (c) $S$. boulardiitreated clarithromycin (CLA) group: mild degeneration in the surface epithelium $(\rightarrow)$, moderate inflammatory cell infiltration $\left({ }^{*}\right)$. (d) Saline-treated CLA group: severe degeneration in the surface epithelium $(\rightarrow)$, severe inflammatory cell infiltration $\left(^{*}\right)$. Haematoxylin and eosin staining, original magnification $\times 100$ (A colour version of this figure can be found online at http://www.journals.cambridge.org/bjn).

elevation of transaminases and cholestatic liver injury are wellknown in patients treated with this antibiotic ${ }^{(10)}$. An earlier study in rats has suggested that hepatocellular injury induced by another macrolide antibiotic, troleandomycin, involved the local depletion of GSH via toxic metabolites formed in cytochrome $\mathrm{P} 450^{(25)}$. According to a meta-analysis by Johnston et al. ${ }^{(26)}$, a dose of 5-40 billion colony-forming units/d of $S$. boulardii had the most promise of decreasing AAD. In accordance with this report, the present findings demonstrate that CLA and MTX increased the intestinal transit, and the drug-induced ileal and hepatic oxidative injury was accompanied by a depletion of hepatic and intestinal GSH stores. Furthermore, $S$. boulardii (approximately in a dose of 2.5 billion colonyforming units/d) effectively reversed all these alterations and normalised the intestinal motility. Apart from its beneficial effects on the prevention of AAD in human subjects, including regimens containing $\mathrm{CLA}^{(27)}, S$. boulardii has not been investigated before for its anti-inflammatory effect on antibiotic-induced gut inflammation. The present results demonstrate that in two different models of drug-induced oxidative injury of the liver and the intestine, $S$. boulardii showed anti-inflammatory and antioxidant actions. Regarding the beneficial effects of probiotics in experimental colitis, Lactobacillus salivarius treatment resulted in decreased MPO activity and increased GSH content in the colon together with reductions in TNF- $\alpha$ and leukotriene B4 levels ${ }^{(28)}$. Various studies investigated the mechanism to explain the anti-inflammatory effects of $S$. boulardii. In vivo and in vitro extracellular signal-regulated kinases $1 / 2$ mitogen-activated protein kinase activity and IL-8 production were shown to decrease with $S$. boulardii treatment in Clostridium difficile toxin A-induced enteritis ${ }^{(29)}$. The anti-inflammatory action of the yeast was further intensified by increasing the barrier integrity of Shigella-infected tissues, thus preventing the migration of pro-inflammatory factors ${ }^{(30)}$. Additionally, recent data showed that the yeast produces a low-molecular weight soluble factor that blocks $\mathrm{NF}-\kappa \mathrm{B}$ activation and NF- $\mathrm{BB}-$ mediated $I L-8$ gene expression in intestinal epithelial cells and monocytes ${ }^{(31)}$.

For many chemotherapeutic agents, including MTX, chemotherapy-induced gut toxicity remains a major dose-limiting side effect, which may limit the efficacy of chemotherapy, affect overall malnutrition, aggravate cancer cachexia and may even contribute to worsened prognosis ${ }^{(32)}$. Patients undergoing chemotherapy experience symptoms of nausea, vomiting, cramping, diarrhoea, abdominal pain and, in its

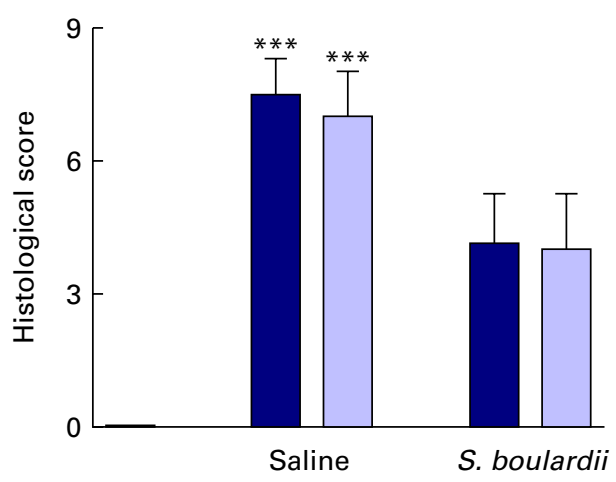

Fig. 6. Histological scores of ileal damage of Saccharomyces boulardiior saline-treated rats that received either methotrexate $(\square)$ injection or daily clarithromycin $(\square)$ by oral administration. Values are means, with standard errors represented by vertical bars. ${ }^{* * *}$ Mean value was significantly different compared with the saline-treated control $(\square$ group $(P<0.001)$. † Mean value was significantly different compared with the respective saline-treated. Mean vaule group $(P<0.05)$. (A colour version of this figure can be found online at http://www.journals.cambridge.org/bjn). 
most severe form, necrosis and perforation of the bowel ${ }^{(33)}$. Despite several symptomatic treatment options in present practice, there is still an obvious requirement to develop new agents to protect the bowel during cancer chemotherapy. $S$. boulardii was shown to significantly suppress expression of pro-inflammatory cytokine genes ${ }^{(34)}$ and reduced diarrhoea, weight loss, leucocyte migration and mucosal inflammation in chemotherapy-induced mucosal damage ${ }^{(35)}$. Consequently, mucositis shown in various models as well as MTX mucositis in the present study were alleviated by $S$. boulardii; therefore, further evaluation is warranted for the use of the yeast in chemotherapy-induced intestinal injury in human subjects.

Probiotics have been demonstrated to support intestinal epithelial barrier function by preventing the adherence of pathogens to the mucosal surface ${ }^{(36)}$ and by enhancing phagocytosis and non-specific immune response ${ }^{(37)}$. Previously, Streptococcus thermophilus was shown to diminish the severity of small-intestinal mucositis in MTX-treated rats, as monitored by the ${ }^{13} \mathrm{C}$-sucrose breath test, where increased tissue MPO activity was also depressed by the probiotic ${ }^{(38)}$. We found increased MPO activity in both the ileal and hepatic tissues of the rats treated with MTX, while $S$. boulardii treatment decreased the MPO levels in the ileum and liver, suggesting decreased neutrophil infiltration to the inflamed tissues. Supporting our findings, an earlier study has shown reduced transepithelial migration of polymorphonuclear leukocytes in the presence of the yeast ${ }^{(30)}$. Regarding the hepatic effects of $S$. boulardii on the MTX-treated group, the increased GSH levels, as compared with the saline-treated MTX group, have not been described before. This finding points to the antioxidant effects of $S$. boulardii on the liver.

The anti-inflammatory effects of $S$. boulardii, as shown in the present study, constitute a growing interest in the nutrition arena. Trials in a wide range of animal models are currently being conducted to identify which component of $S$. boulardii works to reduce the inflammatory status. Whether the live yeast cell per se or the wall structure of the killed yeast is responsible for the beneficial effects is a matter of debate. The study by Generoso et al. ${ }^{(39)}$ showed similar effects with viable or heat-killed $S$. boulardii on preventing bacterial translocation and increasing the anti-inflammatory cytokine, IL-10, levels in rats having intestinal injury. By contrast, Zanello et al. ${ }^{(40)}$ reported that viable and killed $S$. cerevisiae, but not viable $S$. boulardii, was effective in protecting against intestinal inflammation induced by enterotoxigenic Escherichia coli. Even more conflicting were the results by Jawhara et $a l^{(41)}$ stating that the $\beta$-glucan fraction of any yeast ( $S$. cerevisiae, $S$. boulardii or even Candida albicans) has protective effects against intestinal inflammation in mice.

CLA is an antibiotic that might cause altered gut flora more than MTX. Altered gut microbiota is thought to cause chronic liver injury via bacterial translocation through the permeable tight junctions of the gut into the portal bloodstream. Since the data from other animal models have shown that translocation from the gut to the liver axis is reduced with $S$. boulardii treatment ${ }^{(42,43)}$, this is likely to be a mechanism for the decreased inflammation with the probiotic treatment. Probiotic administration appears to decrease both the hepatic and ileal MDA levels in the CLA-treated group. However, the mechanism of liver damage is totally different in the MTX group, which is apparent in the failure of the probiotic to depress hepatic MDA levels, suggesting that the probiotic may not be fully effective against drug-related hepatotoxicity.

In conclusion, our findings suggest that $S$. boulardii effectively suppressed the oxidative damage in rats due to MTXor CLA-induced inflammation in the ileum and liver. These results are confirmed by the alleviated histological scores in the gut when $S$. boulardii was added to the treatment with a pro-inflammatory antibiotic or chemotherapeutic agent. The present results suggest that antioxidant protection is another mechanism through which $S$. boulardii is beneficial in the prevention of $\mathrm{AAD}$. Moreover, the effects of that probiotic on MTX-induced toxicity is a novel finding proposing $S$. boulardii as an adjunct to clinical regimens in an attempt to prevent the intestinal and, in part, hepatic side effects.

\section{Acknowledgements}

No funding has been received for the study. The contributions of authors were as follows: D. G. D., F. E. and B. C. Y. participated in the design of the study. D. G. D., Z. N. O. K. and B. C. Y. conducted the data analysis. F. E. carried out the histological scoring of the ileum tissues. D. G. D., Z. N. O. K. and B. C. Y. wrote the draft of the final manuscript. All authors carried out the search strategy, took part in the revision of the manuscript, and read and approved the final manuscript. The authors have no conflicts of interest to declare.

\section{References}

1. Dalmasso G, Cottrez F, Imbert V, et al. (2006) Saccharomyces boulardii inhibits inflammatory bowel disease by trapping $\mathrm{T}$ cells in mesenteric lymph nodes. Gastroenterology 131, $1812-1825$.

2. Hempel S, Newberry SJ \& Maher AR (2012) Probiotics for the prevention and treatment of antibiotic-associated diarrhea: a systematic review and meta-analysis. JAMA 307, 1959-1969.

3. Buts JP, De Keyser N \& Raedemaeker L (1994) Saccharomyces boulardii enhances rat intestinal enzyme expression by endoluminal release of polyamines. Pediatr Res $\mathbf{3 6}$, 522-527.

4. Buts JP, Bernasconi P, Vaerman JP, et al. (1990) Stimulation of secretory Ig A and secretory component of immunoglobulins in small intestine of rats treated with Saccharomyces boulardii. Dig Dis Sci 35, 251-256.

5. Pothoulakis C (2009) Review article: anti-inflammatory mechanisms of action of Saccharomyces boulardii. Aliment Pharmacol Ther 15, 826-833.

6. McFarland LV (2010) Systematic review and meta-analysis of Saccharomyces boulardii in adult patients. World $J$ Gastroenterol 14, 2202-2222.

7. Bartlett JG (2002) Clinical practice. Antibiotic-associated diarrhea. $N$ Engl J Med 346, 334-339.

8. Drehobl MA, De Salvo MC, Lewis DE, et al. (2005) Singledose azithromycin microspheres vs clarithromycin extended release for the treatment of mild-to-moderate communityacquired pneumonia in adults. Chest 128, 2230-2237. 
9. McKenna S \& Evans G (2001) Macrolides: a Canadian Infectious Disease Society position paper. Can J Infect Dis 12, 218-231.

10. Leitner JM, Graninger W \& Thalhammer F (2010) Hepatotoxicity of antibacterials: pathomechanisms and clinical. Infection 38, 3-11.

11. Olivet J, Cowan KH \& Curt GA (1983) The pharmacology and clinical use of methotrexate. $N$ Engl J Med 309, 1094-1104.

12. Curd CD, Manno JE \& Stewart JJ (1985) Effects of methotrexate on intestinal transit in rats. Fundam Appl Toxicol 5, 991-996.

13. Jahovic N, Sener G, Cevik H, et al. (2004) Amelioration of methotrexate-induced enteritis by melatonin in rats. Cell Biochem Funct 22, 169-178.

14. Buts JP, Bernasconi P \& Craynest MPV (1986) Response of human and rat small intestinal mucosa to oral administration of Saccharomyces boulardii. Pediatr Res 20, 192-196.

15. Blehaut H, Massot J \& Elmer GW (1989) Disposition kinetics of Saccharomyces boulardii in man and rat. Biopharm Drug Dispos 10, 353-364.

16. Kim YC, Lee JH, Kim SH, et al. (2005) Effect of CYP3A1(23) induction on clarithromycin pharmacokinetics in rats with diabetes mellitus. Antimicrob Agents Chemother 49, $2528-2532$.

17. Udassin R, Eimerl D, Schiffman J, et al. (1994) Epidural anesthesia accelerates the recovery of postischemic bowel motility in the rat. Anesthesiology 80, 832-836.

18. Bradley PP, Priebat DA, Christersen RD, et al. (1982) Measurement of cutaneous inflammation. Estimation of neutrophil content with an enzyme marker. $J$ Invest Dermatol 78, 206-209.

19. Aykaç G, Uysal M, Yalçin AS, et al. (1985) The effect of chronic ethanol ingestion on hepatic lipid peroxide, glutathione, glutathione peroxidase and glutathione transferase in rats. Toxicology 36, 71-76.

20. Casini AF, Ferrali M, Pompella A, et al. (1986) Lipid peroxidation and cellular damage in extrahepatic tissues of bromobenzene-intoxicated mice. Am J Pathol 123, 520-531.

21. Sener G, Eksioglu-Demiralp E, Cetiner M, et al. (2006) L-Carnitine ameliorates methotrexate-induced oxidative organ injury and inhibits leukocyte death. Cell Biol Toxicol 22, 47-60.

22. Peeters TL (1993) Erythromycin and other macrolides as prokinetic agents. Gastroenterology 105, 1886-1899.

23. Bortolotti M, Mari C, Brunelli F, et al. (1999) Effect of intravenous clarithromycin on interdigestive gastroduodenal motility of patients with functional dyspepsia and Helicobacter pylori gastritis. Dig Dis Sci 44, 2439-2442.

24. Rodrigues AD, Roberts EM, Mulford DJ, et al. (1997) Oxidative metabolism of clarithromycin in the presence of human liver microsomes. Major role for the cytochrome P4503A (CYP3A) subfamily. Drug Metab Dispos 25, 623-630.

25. Pessayre D, Tinel M, Larrey $\mathrm{D}$, et al. (1983) Inactivation of cytochrome P-450 by a troleandomycin metabolite. Protective role of glutathione. J Pharmacol Exp Ther 224, 685-691.

26. Johnston BC, Supina AL, Ospina M, et al. (2007) Probiotics for the prevention of pediatric antibiotic-associated diarrhea. Cochrane Database of Systematic Reviews, issue 2 CD004827.

27. Duman DG, Bor S, Ozutemiz O, et al. (2005) Efficacy and safety of Saccharomyces boulardii in prevention of antibiotic-associated diarrhoea due to Helicobacter pylori eradication. Eur J Gastroenterol Hepatol 17, 1357-1361.

28. Peran L, Camuesco D, Comalada M, et al. (2005) Preventative effects of a probiotic, Lactobacillus salivarius ssp. salivarius, in the TNBS model of rat colitis. World $J$ Gastroenterol 11, 5185-5192.

29. Chen X, Kokkotou EG, Mustafa N, et al. (2006) Saccharomyces boulardii inhibits ERK1/2 mitogen-activated protein kinase activation both in vitro and in vivo and protects against Clostridium difficile toxin A-induced enteritis. J Biol Chem 281, 24449-24454.

30. Mumy KL, Chen X, Kelly CP, et al. (2008) Saccharomyces boulardii interferes with Shigella pathogenesis by postinvasion signaling events. Am J Physiol Gastrointest Liver Physiol 294, G599-G609.

31. Sougioultzis S, Simeonidis S, Bhaskar KR, et al. (2006) Saccharomyces boulardii produces a soluble antiinflammatory factor that inhibits NF-kappaB-mediated IL-8 gene expression. Biochem Biophys Res Commun 343, 69-76.

32. Xue H, Sawyer MB, Wischmeyer PE, et al. (2011) Nutrition modulation of gastrointestinal toxicity related to cancer chemotherapy: from preclinical findings to clinical strategy. JPEN J Parenter Enteral Nutr 35, 74-90.

33. Sonis ST, Elting LS, Keefe DMK, et al. (2004) Perspectives on cancer therapy-induced mucosal injury: pathogenesis, measurement, epidemiology, and consequences for patients. Cancer 100, 1995-2025.

34. Lee SK, Kim YW, Chi SG, et al. (2009) The effect of Saccharomyces boulardii on human colon cells and inflammation in rats with trinitrobenzene sulfonic acid-induced colitis. Dig Dis Sci 54, 255-263.

35. Sezer A, Usta U \& Cicin I (2009) The effect of Saccharomyces boulardii on reducing irinotecan-induced intestinal mucositis and diarrhea. Med Oncol 26, 350-357.

36. Forestier C, De Champs C \& Vatoux C (2001) Probiotic activities of Lactobacillus casei rhamnosus: in vitro adherence to intestinal cells and antimicrobial properties. Res Microbiol 152, 167-173

37. Isolauri E (2001) Probiotics in human disease. Am J Clin Nutr 73, $1142 \mathrm{~S}-1146 \mathrm{~S}$

38. Tooley KL, Howarth GS, Lymn KA, et al. (2006) Oral ingestion of Streptococcus thermophilus diminishes severity of small intestinal mucositis in methotrexate treated rats. Cancer Biol Ther 5, 593-600.

39. Generoso SV, Viana ML, Santos RG, et al. (2011) Protection against increased intestinal permeability and bacterial translocation induced by intestinal obstruction in mice treated with viable and heat-killed Saccharomyces boulardii. Eur J Nutr 50, 261-269.

40. Zanello G, Meurens F, Berri M, et al. (2011) Saccharomyces cerevisiae decreases inflammatory responses induced by $\mathrm{F}^{+}$enterotoxigenic Escherichia coli in porcine intestinal epithelial cells. Vet Immunol Immunopathol 141, 133-138.

41. Jawhara S, Habib K, Maggiotto F, et al. (2012) Modulation of intestinal inflammation by yeasts and cell wall extracts: strain dependence and unexpected anti-inflammatory role of glucan fractions. PLoS One 7, e40648.

42. Lessard M, Dupuis M, Gagnon N, et al. (2009) Administration of Pediococcus acidilactici or Saccharomyces cerevisiae boulardii modulates development of porcine mucosal immunity and reduces intestinal bacterial translocation after Escherichia coli challenge. J Anim Sci 87, 922-934.

43. Akyol S, Mas MR, Comert B, et al. (2003) The effect of antibiotic and probiotic combination therapy on secondary pancreatic infections and oxidative stress parameters in experimental acute necrotizing pancreatitis. Pancreas 26, $363-367$. 